\title{
Modifications of the Poggendorff effect as a function of random dot textures between the verticals
}

\author{
ROBERTO MASINI, TOMMASO COSTA, MARIO FERRARO, and ANGELO DE MARCO \\ University of Turin, Turin, Italy
}

\begin{abstract}
In the present research, we investigated the modification of the strength of the Poggendorff illusion as a function of different densities of random dot textures filling the space between the verticals. The results of Experiment 1 show that the illusory effect is a nonlinear function of the texture parameter $r$, the ratio of black pixels to white and black pixels, with a minimum for $r=$ 0.5 , approximately, and a maximum for $r=0$ and $r=1$. The results may be interpreted by an analytical model of perceptual space dynamics, in which the effect depends on the amount of interaction between points of different light intensity. A computer simulation performed by applying the analytical model to different values of $r$ shows a good agreement between the predictions and the experimental data. To test the hypothesis underlying the model, a second experiment was carried out to measure the magnitude of the expansion of the space between the verticals as a function of the parameter $r$. The results are consistent with the hypothesis of the model. The overall data are discussed in terms of their implications on various theories proposed for the Poggendorff illusion.
\end{abstract}

In the original version of the Poggendorff illusion, two colinear obliques separated by a pair of verticals appear displaced from their physical alignment (see Figure 1). The various explanations proposed for this effect can be grouped under four main categories, based on (1) structural factors, (2) depth processing of parts of the figure, (3) mistracking and angular induction, and (4) perceptual modifications of the space between the parallels. In the first case, the illusory effect is supposed to depend on distortions brought about by the structural properties of the optical or neural systems; mechanisms such as optical blur and lateral inhibition could induce distortions in the region of the intersections between lines-for example, expansion of acute angles (Chiang, 1968; Glass, 1970; Helmholtz, 1886; Hotopf \& Ollerearnshaw, 1972a, 1972b; Hotopf, Ollerearnshaw \& Brown, 1974; Stuart \& Day, 1980). Yet the illusion is still present when these factors are removed (Day, Dickinson, \& Jory, 1977; Goldstein \& Weintraub, 1972; Gregory, 1972; Schiller \& Weiner, 1972; Springbett, 1961). The second category of explanation attributes it to an inappropriate application of constancy scaling mechanisms to bidimensional patterns (Gillam, 1971, 1980, 1981; Green \& Hoyle, 1963, 1964; Thiery, 1896). The presence of the illusory effect in the so-called bisection forms (Day, 1989; Day \& Kasperczyk, 1985), lack-

This research was partly supported by Grant 91.03842.CT08 from Consiglio Nazionale delle Ricerche. Portions of this research were presented at the 1992 15th European Conference on Visual Perception. Correspondence concerning this article should be sent to $\mathrm{R}$. Masini, Department of Psychology, University of Turin, via Po, 14, 10123 Turin, Italy.

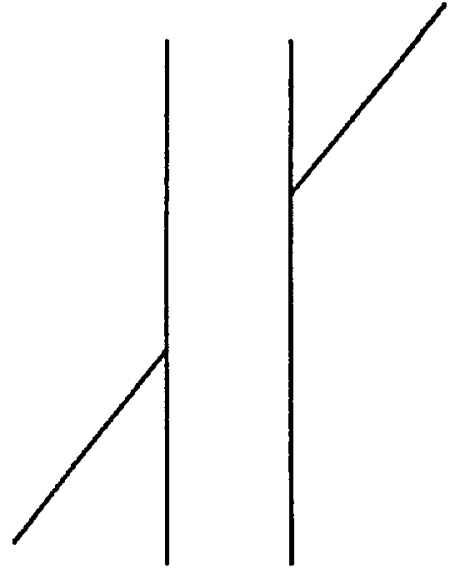

Figure 1. The Poggendorff illusion. The upper right oblique appears too high to be aligned with the prolongation of the lower left oblique.

ing depth cues, seems to contrast this interpretation. More recently, Parks and Hui (1989) showed that a pictorial difference in height is not a necessary and sufficient cause for the illusion.

A third, more plausible type of explanation argues, in general, for a misprojection of the projected path of an oblique across the space to the opposite vertical. This interpretation has the advantage of explaining the Poggendorff illusion in terms of alignment or position without referring to other perceptual dimensions. According to Tong and Weintraub (1974), the actual intersection between the oblique and vertical is the prime requisite for 
the Poggendorff effect, as shown both by the decline of the error when the intersection is degraded or removed and by the fact that the amount of misprojection is a constant angle, whatever the distance between the oblique and the target line (Tong \& Weintraub, 1974; Weintraub \& Krantz, 1971). The hypothesis is that the intersection produces cognitive (higher order) mistracking. To account for some anomalies that remain unexplained by the expansion of acute angles (e.g., Gillam's right-angle Poggendorff effect, the Loeb illusion), Hotopf (1981) proposed a common feature named the alignment displacement effect (ADE). In this case, a line (pointer) aligned with another line (target), which is one of a pair of lines, will appear displaced in the direction of the second line (induction). As for the mechanism underlying the $\mathrm{ADE}$, Hotopf, like Tong and Weintraub, excludes a neural interaction and argues for mistracking where the eye movement is dispatched at a fixed angle following a cognitive program. The program setup depends on locating the target, the orientation of the induction, and the angle at which the pointer stands. More recently, Greene and co-workers proposed the term angular induction for the process by which the presence of one or more lines can affect the perceived angle of another line or its projection. The experimental data from these authors showed that, by using long-line stimuli, both proximal and distal lines contribute to the induction process (Greene, 1987; Greene \& Pavlov, 1989); this influence is in turn determined by the angle between each induction line and the oblique, and these effects can be summed independently. Furthermore, within each induction line, it is possible to show an interaction of both global and local processes. As for the mechanism responsible for the induction process, Greene and AlQuaddoomi (1990), keeping in mind hypothetical models of neural interaction (Tyler \& Nakayama, 1984), appear to be cautious in extending them to the global induction process, concluding that "an alternative concept, such as distortion of the spatial field, may be appropriate" (p. 252). These types of explanations will be taken up later, in the discussion of our own data.

The fourth type of explanation is directly related to our present research. In this case, the Poggendorff illusion is considered more an illusion of linear extent than an illusion of direction, because the misalignment derives from a distortion of the space between the verticals. This distortion has been explained in different ways. Some authors (Greist-Bousquet \& Schiffman, 1981, 1985, 1986; GreistBousquet, Schiffman, Dorsett, \& Davis, 1989; Judd, 1899; Pressey, 1971; Pressey \& Sweeney, 1972; Wilson \& Pressey, 1976) argue for the presence in the Poggendorff configuration of some of the components of the Müller-Lyer illusion, which in turn cause the underestimation of the oblique extent between the verticals. This interpretation seems to be supported by experimental results showing a significant correlation between alignment judgments and extent judgments (Greist-Bousquet et al., 1989). But the above findings are contrasted by the data of experiments showing an accurate estimation of oblique and right-angle extents (Day, Jolly, \& Duffy, 1987), or even an overestimation of the oblique extent (Finlay \& Caelli, 1975; Wilson \& Pressey, 1976).

As an alternative approach, a smaller number of authors consider the contraction of the right-angle extent between the two verticals to be fundamental. Day and Dickinson (1976) explained the misalignment in terms of three basic components present in the configuration: the horizontalvertical, the longitudinal transverse, and obtuse angle effects. These effects singly or jointly can give rise to an apparent elongation-contraction of the space between the colinear elements. In this sense, the illusion is explained on the basis of other illusions (the vertical illusion, the rectangles illusion). Zanuttini (1976), instead, hypothesized a shrinkage of the surface on which the oblique lines, considered as a unit, are amodally completed; this shrinkage in turn causes the displacement of the visible parts of the oblique line. Contrary to Day and Dickinson, it is not the visible space that shrinks, but the amodal space. Other authors (De Marco, Magnetti, \& Saroldi, 1991; Quina \& Pollack, 1973; Quina-Holland, 1977) have provided experimental evidence of a contours interaction, which causes the contraction or the enlargement of the visible space between the verticals; however, they did not present a definite explanatory mechanism.

The present experiments originated from the consideration of a field model of visual illusions. Following Eriksson (1970), all perceptual elements influence each other according to a law of interaction, $f=f(d)$, where $d$ is the distance between the elements, and it is usually assumed that a positive $f(d)$ corresponds to an attraction and a negative $f(d)$ corresponds to a repulsion between the perceptual elements; in general, visual interactions affect the perception of distances. The principle of additivity is postulated; the linearity of the process is assumed. In this approach, the strength of the Poggendorff illusion depends on a contraction of the space, $S$, between the parallels, caused in turn by attraction forces in $S$. The main problem of field theories is the lack of an analytical definition of the potential's form. The potential can, in fact, change from attraction to repulsion or vice versa, if more than one geometric element is present in the perceptual space $S$.

From a phenomenological point of view, we can consider the Poggendorff illusion as an instance of the filledunfilled space effect, the Oppel-Kundt illusion (see Figure 2). In the case of the unfilled space, the attraction forces prevail; we can see an underestimation of the distance. Instead, by adding other points between the endpoints, a repulsion force appears between the near points, giving rise to an enlargement of the space. If this hypothesis is truly consistent, filling the space between the ver-

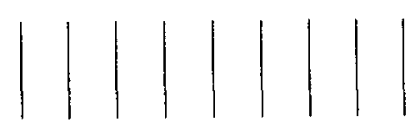

Figure 2. The Oppel-Kundt illusion. The filled space appears longer than the unfilled space. 
ticals of the Poggendorff illusion with random dot textures of different density could modify the strength of the illusion according to a function that must be specified. Furthermore, there are data in the literature supporting a relationship between texture density and perceived magnitude of a surface (Bazzeo \& Zanuttini, 1978; Newman, 1973), even though the relationship between these variables is not simple. In 1993, Bazzeo, Vicario, and Zambianchi conducted an experiment specifically to determine the relationship between the strength of the Poggendorff illusion and the presence of a texture between the verticals. Using regular textures and a magnitude estimation method to determine the apparent expansion of the surfaces and the strength of the illusion, they found that an increase in apparent linear extent within the parallels corresponds to a significant decrease in the magnitude of the illusion. They explained the Poggendorff effect as an instance of the Oppel-Kundt illusion, but did not supply a unified explanatory model of the space dynamics. In a recent work, Masini, Sciaky, and Pascarella (1992) investigated the strength of the illusory effect as a function of the different orientation of a parallel-line texture between the verticals. The results showed that the effect was a linear function of the progressive discrepancy between the angle of the lines of the texture and that of the obliques. These results have been interpreted within the "perceptual compromise"' hypothesis (Day et al., 1987; Day \& Kasperczyk, 1985), but stress the role of the apparent elongation of the vertical extension.

Summing the above considerations led us to hypothesize that elements forming texture or, in general, elements of different light intensity, can give rise to repulsive interactions contrasting the illusory effect. We have assumed that the interaction between two points $p$ and $q$ in the visual space $S$ can be modeled by a function of the type

$$
f(p, q)=I_{0}-|I(p)-I(q)| g(p, q),
$$

where $I_{0}$ is a constant, $I(p), I(q)$ are light intensities at the points $p$ and $q$, respectively, and $g$ is a positive-valued, decreasing function of $d(p, q)$, the distance between $p$ and $q$. From Equation 1 it follows that two points with the same light intensity are affected by an interaction $f(p, q)$ $=I_{0}$, whereas if they have different intensity, the interaction is decreased by a factor $|I(p)-I(q)| g(p, q)$. The global interaction among points in $S$ is given by

$$
f=\sum_{p \neq q} f(p, q)
$$

This model allows us to formulate some simple predictions concerning the strength of the Poggendorff illusion as a function of the light intensity in $S$, the space between the verticals. Suppose that $S$ is a discrete grid, and further suppose that pixels can be only black or white, that is, $I=0$ or $I=1$. Define $r=n(b) /[n(b)+n(w)]$, where $n(b)$ and $n(w)$ are the numbers of black and white pixels in $S$. The interaction is maximum when $r=1$ or $r=0$, and minimum when $r=0.5$ (cf. Equations 1 and 2 ). Then, the perceptual space $S$ should increase for $0 \leq$ $r<0.5$ and should start decreasing again for $0.5<r \leq$ 1. Correspondingly, the strength of the illusion should decrease for $0 \leq r<0.5$ and increase for $0.5<r \leq 1$, with a minimum at $r=0.5$. The predictions of this model have been investigated in an experiment in which the space between the verticals was filled with a random dot texture of different values of $r$, from 0 to 1 , at steps of about 0.12 . We do not claim here that the expansion of the space between the verticals causes the Poggendorff illusion, but merely that the effect is influenced by this expansion; indeed, it has been suggested (see Coren \& Girgus, 1978) that the Poggendorff illusion can depend on multiple determinants.

\section{EXPERIMENT 1}

The purpose of Experiment 1 was to measure the magnitude of the Poggendorff effect as a function of the texture parameter $r$, the ratio of black pixels to white and black pixels. According to our hypothesis, the magnitude of the illusion should be minimum for $r=0.5$ and maximum for $r=0$ and $r=1$. Nine different textures have been evaluated, with $r$ ranging from 0 to 1 at steps of about 0.12 .

\section{Method}

Subjects. In this experiment we used a within-subjects design. Eight students participated in the experiment; they were all volunteer undergraduates, naive to the experimental aims, and had normal visual acuity. Their average age was 21 years.

Apparatus. The stimulus patterns were generated on a personal computer (PC486) with VGA card, presented at the center of the screen in a frontoparallel plane; the viewing distance was about $70 \mathrm{~cm}$. The screen contrast and the illumination within the room were kept constant throughout the experiment. The subjects were instructed to keep their heads upright. The computer was programmed to present, in each trial, the experimental pattern with the right oblique segment randomly displaced at the top or the bottom of the right vertical line. The subjects moved the right oblique segment up and down until they thought it was correctiy aligned with the left one. They did this by pressing four different keys. Two keys allowed them to move the segment up and down 5 pixels per keystroke; the other two keys allowed movement of 1 pixel per keystroke. The same program recorded the alignment error; the error was expressed in pixels and the value was positive when the right oblique segment was lower than the correct alignment (presence of the illusory effect), and negative in the other case.

Materials. The basic pattern was the standard form of the Poggendorff illusion, black on a white foreground (see Figure 1), with the vertical lines $7.8 \mathrm{~cm}$ long, separated from each other by $1.5 \mathrm{~cm}$, and the two oblique segments $3.2 \mathrm{~cm}$ long. All the lines were 1 pixel thick, corresponding to $0.33 \mathrm{~mm}$. The angle formed by the obliques and the vertical lines was $45^{\circ}$. The space between the verticals was unfilled, the ratio, $r$, of black pixels to white and black pixels was $r=0$. The nine experimental conditions were obtained by randomly changing some white pixels to black, with different values of the parameter $r$, from $r=0$ (unfilled space) to $r=1$, at steps of 0.12 . The position of the texture elements was randomly generated by a uniform probability distribution. This distribution, with the same $r$ value, was generated at every single trial.

Procedure. We used the adjustment method to determine the mean alignment error. Each trial consisted of moving the right oblique segment up and down along the right vertical line, starting from 
a randomly assigned position at the top or bottom of that line. The subjects' task was to align it with the left oblique segment. The subjects performed 20 trials each, for each experimental condition. At each trial, the texture, with fixed $r$, was randomly generated. There were no time limits for adjustments, and the subjects proceeded at their own pace. After an adjustment had been completed, they moved on to the next stimulus by pressing a key. In order to prevent the position effect, we partly counterbalanced the presentation order of the patterns within subjects.

\section{Results and Discussion}

The results of Experiment 1 are shown in Figure 4, where the magnitude of the illusion is plotted as a function of the parameter $r$. A one-way analysis of variance with repeated measures shows that the texture factor significantly influenced the strength of the illusion $[F(7,71)=$ $7.798, p<.0001]$.

The strength of the illusion is a nonlinear function of $r$ (see Figure 4). A quadratic fit, $s=a+b r+c r^{2}$, performed by using the standard least squares method, yielded reduced $\chi^{2}(6)=0.13$ and $\alpha>.85$, with $a=$ $4.56, b=-4.5$, and $c=4.18$. Our data suggest that the illusory effect is a parabolic function of the density of the textures filling the space between the verticals. This
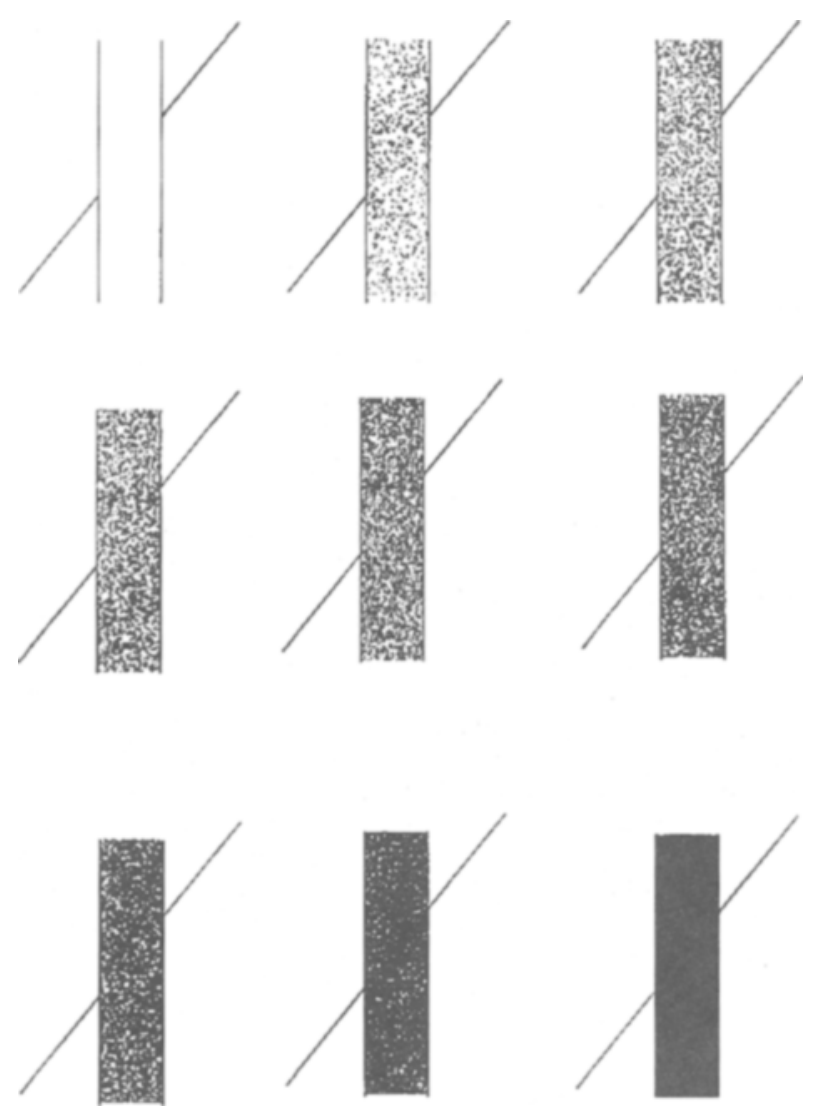

Figure 3. The nine experimental patterns. The space between the verticals is changed from white, $r=0$, to black, $r=1$, at steps $d r=$ 0.12.

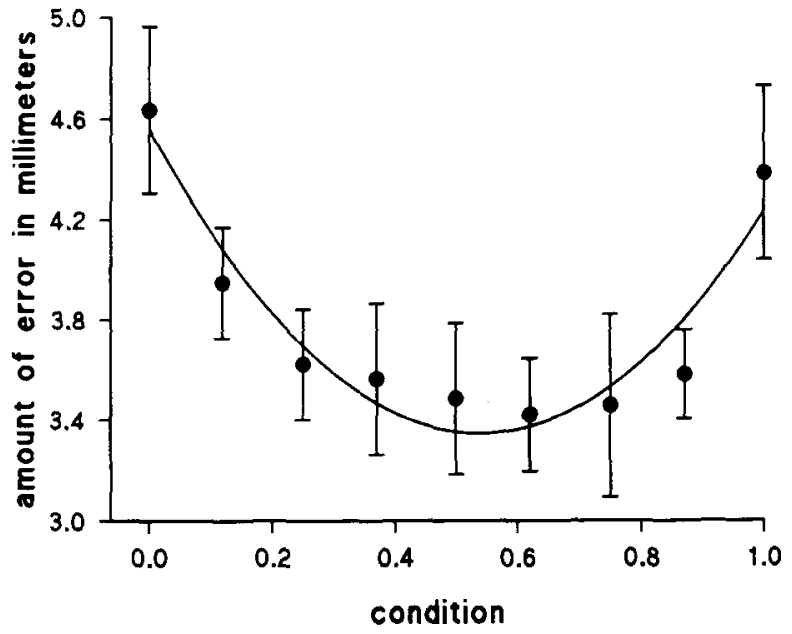

Figure 4. Strength of the illusion as a function of $r$. Number of trials per point, 160 . The solid curve is the result of a quadratic fit.

function has a minimum of about $r=0.5$. This means that the effect is lowest when there are equal numbers of black and white pixels. On the other hand, the effect is maximal for $r=0$ and $r=1$ with a little asymmetry between the two conditions. These results are consistent with the hypothesis that the elements forming texture can give rise to repulsive interactions contrasting the illusory effect.

This hypothesis can be further tested by using Formulas 1 and 2 to simulate the strength of the illusory effect for different values of $r$. The experimental setup was simulated by a matrix of 0 and 1 , randomly generated so that $I(i, j)=0$ or 1 , and the function $g$ was defined as

$$
g[d(p, q)]=\left\{\begin{array}{l}
1 \text { if } q \text { belongs to a 4-connected } \\
\text { neighborhood of } p \\
0 \text { otherwise. }
\end{array}\right.
$$

Thus, any single pixel interacted only with the elements of its nearest neighborhood. We have assumed that the strength of the effect is a linear function, $s=a+b f$, of the total interaction $f$ in the space $S$, where $a$ and $b$ are parameters that have been determined by minimizing the $\chi^{2}$ function. Experimental and predicted data are shown in Figure 5; in this case, $a=2.3$ and $b=0.27$ $10^{-4}$, reduced $\chi^{2}(6)=0.96, \alpha>.4$. The results show a good agreement between the predictions derived from the analytical model and the experimental data. It is easy to show that these results do not depend on the particular form of the function $g(d)$.

\section{EXPERIMENT 2}

The basic hypothesis of our model is that the random dot textures cause an apparent expansion of the space $S$ between the verticals; this hypothesis is supported, as mentioned in the introduction, by some experimental evidence in the case of regular textures (Bazzeo et al., 1993; Bazzeo \& Zanuttini, 1978). We further tested this hypoth- 
esis by carrying out an experiment to measure the magnitude of the expansion of the space between the parallels as a function of the texture parameter $r$, the ratio of black pixels to white and black pixels. On the basis of our model (cf. Formulas 1 and 2), the expansion should be a function of $r$ with a maximum at $r=0.5$. We used the same experimental patterns as in Experiment 1, but removed the two obliques. The width of each experimental pattern was compared with a space where $r=0$.

\section{Method}

Subjects. In Experiment 2, we used a within-subjects design. Nine subjects participated in the experiment; they were all volunteers, naive to the experimental aims, and had normal visual acuity.

Apparatus. The general apparatus was the same as that used in Experiment 1. The computer was programmed to present, in each trial, the following configuration: two vertical parallel lines bounding a random dot texture (standard stimulus), and two other vertical parallel lines bounding an unfilled space (the variable stimulus). The distance between these two stimuli was $3 \mathrm{~cm}$. In order to equate the apparent width of the space between the vertical lines of the two stimuli, the subjects moved the right vertical line of the variable stimulus by pressing a key, 1 pixel per keystroke. The right vertical line of the variable stimulus was randomly positioned around the true position, the position in which the two stimuli had the same distance between the vertical lines. The same program recorded the mean apparent width error, the difference between the subjective and true positions of the right vertical line of the variable pattern; the error was expressed in pixels and the value was positive when the right segment was to the right of the correct alignment (presence of the illusory effect).

Materials. The experimental patterns were constituted by the nine experimental configurations of Experiment 1, after removing the obliques, and by two vertical parallel lines bounding an unfilled space (variable stimulus), placed at either the right or left of the previous configuration. As in Experiment 1, the textures between the verticals of the standard stimulus were obtained by randomly changing some white pixels to black, with different values of the parameter $r$, from $r=0$ (unfilled space) to $r=1$, at steps of 0.12 . The position of the texture elements was randomly generated by

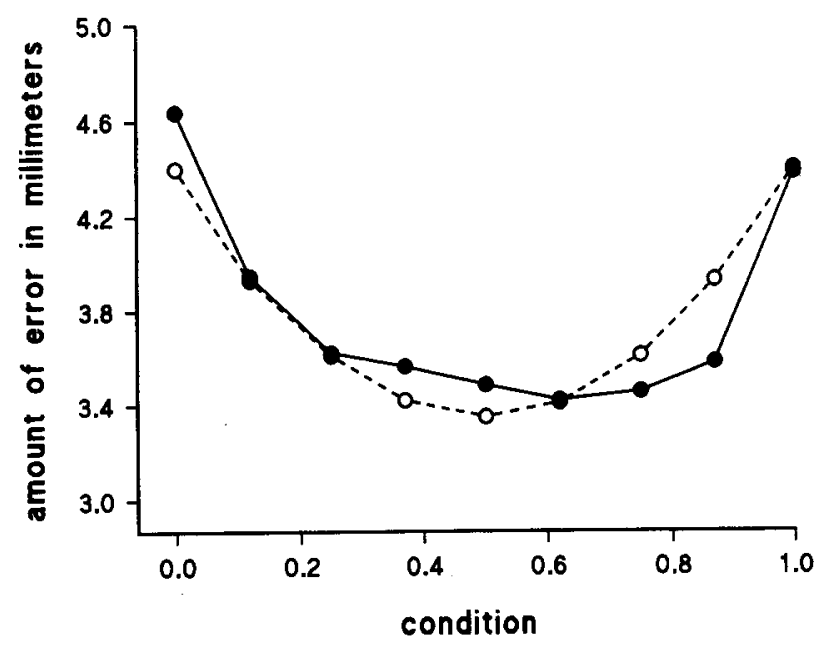

Figure 5. Experimental data (filled circles) and predicted values (open circles) of the strength of the illusion, as derived by means of Formulas 1 and 2.

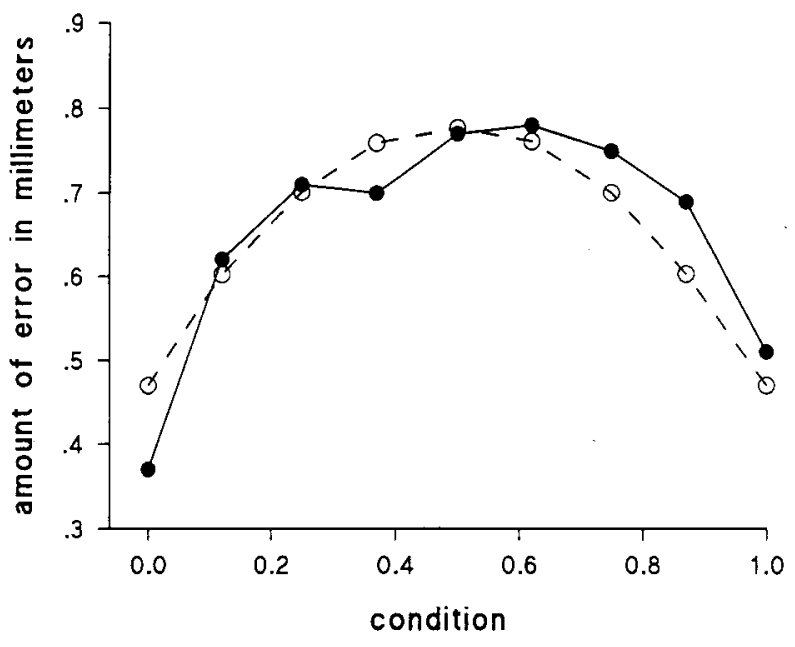

Figure 6. Experimental data (filled circles) and predicted values (open circles) of the strength of the expansion, as derived by means of Formulas 1 and 2.

a uniform probability distribution. This distribution, with the same $r$ value, was generated at every single trial.

Procedure. We used the adjustment method to determine the mean apparent width of the surfaces. Each trial consisted of moving the right vertical line of the variable stimulus toward the right or the left, starting from a random position at either the left or right of that of physical equivalence. The remaining procedure was the same as that in Experiment 1.

\section{Results and Discussion}

The results of Experiment 2 are in agreement with the predictions of our model. Figure 6 shows the comparison between the experimental data and the predicted values; in this case, the parameters of the fit are $a=0.47, b=$ $810^{-6}$, reduced $\chi^{2}(6)=0.7$, and $\alpha>.60$. It is interesting to note that the apparent expansion for $r=0.62$ (i.e., the maximum) is $22 \%$. This is close to the maximal reduction obtained for the Poggendorff illusion, which is $26 \%$.

\section{GENERAL DISCUSSION}

The results of Experiment 1 show that the strength of the Poggendorff illusion is a parabolic function of the density of random dot textures, generated by a uniform distribution, filling the space between the verticals. We have suggested that this effect depends on the amount of interaction between points of different light intensity. Filling the spaces, $S$, with textures of increasing density should produce an increase of the global repulsive interaction among the points, and then should produce an apparent expansion of the space, $S$, between the verticals. The data provided by Experiment 2 lend support to this hypothesis.

We can consider our data with respect to the four main categories of explanation of the Poggendorff illusion discussed in the introduction. Explanations pertaining to structural factors, responsible for the optical blur and lateral inhibition, appear difficult to support because the inter- 
sections between lines of the standard Poggendorff configuration were constant in the experiment. However, as in previous research conducted by Masini et al. (1992), the illusion reduces but does not disappear, and therefore we cannot exclude the possibility that part of the effect is due to structural or other factors. Because the configuration parameters used in the present experiments were the same as those in Masini et al., it is possible to discuss the results together. When only the two obliques were present (Masini et al., 1992), we obtained an error of about $1 \mathrm{~mm}$ (Experiment 2, Condition ST2); this can be considered a general alignment error in the oblique orientation. The contribution, in terms of error, of the presence of the verticals (a constant difference in alignment error between the conditions of Experiments 1 and 2) was about $1 \mathrm{~mm}$. If we subtract these values from the lowest alignment error obtained in the present research $(3.42 \mathrm{~mm})$, we find that about $1.42 \mathrm{~mm}$ is unaccounted for.

The explanations referring to depth processing are questionable because, in the present experiments, differences in the pictorial height of the obliques were kept constant, and only the texture density was changed. However, texture factors modified the effect. On the other hand, because the texture gradient in our patterns is null, the effects due to modification of the surface slant were eliminated. However, the textures could have introduced some other depth effect; for example, they could have facilitated the separation in depth between the obliques and the filled space. In this sense, the figural unification of the obliques could have reduced the misalignment. There is evidence in the literature that a reduction of the interaction between obliques and inducing lines (e.g., the motion of the obliques) reduces the magnitude of the illusion (Benussi, 1914; Mori, 1981).

In general, explanations that argue for a misprojection of the projected path appear to be more coherent with the present experimental data than were previous models. However, we would like to remark that (1) the data supporting this interpretation were prevalently obtained with substantial modifications of the original Poggendorff configuration; (2) sometimes different studies show important discrepancies in the data concerning the same pattern; compare, for example, results from Experiment 3 in Weintraub and Krantz (1971), and from Greene and Pavlov (1989); and (3) as indicated by Hotopf (1981), a direct measurement of eye movement, actual or cognitively programmed, should be required. But despite these objections, our own data can be explained according to these models; that is, the density of the textures could modify, in a differential way, the integrity of the edge of the surface. In this sense, the strength of the induction process should be reduced and, consequently, the strength of the illusion should reduce. At $r=0.5$, where the contrast between the surface and the line is minimum, the effect should be minimum. The action of the texture elements should both interfere with the hypothetical neural processes underlying the induction and modify the figural conditions favoring the detection of the contact line. Lack- ing experimental evidence, this remains a plausible hypothesis, which should permit a falsification of our model, for example, by using local texture (proximal to the contact, or the target, or both) as a modifier of the induction processes.

Some considerations can be made regarding the explanations that argue for a distortion of the space between the verticals as a basis for the Poggendorff illusion. First, the model referring to Müller-Lyer components cannot fully explain our data because these components are still present in our configurations. Furthermore, our results are opposite to what can be expected from an interpretation based on amodai completion; in fact, increasing the density of the textures should diminish the energy available in the amodal space and thus increase the shrinkage of the segment constituted by the obliques. This effect should therefore give rise to an increase of the illusory effect, but our data show the opposite. As for Day and Dickinson's (1976) explanation, increasing the texture density should counterbalance both the horizontal-vertical $(\mathrm{H}-\mathrm{V})$ and the longitudinal-transverse (L-T) effects; however, Day and Dickinson did not formulate a quantitative model. In conclusion, we think that our data are better explained in terms of a field model of space dynamics.

It could be argued that, because $r=0.5$ corresponds to the maximal connectivity between the dots, a subjective contour connecting through the texture could reduce the strength of the illusion. However, it must be observed that this explanation contrasts the results obtained by Bazzeo et al. (1993). The textures they used were constituted by various types of elements (black patches, horizontal and vertical lines), some of which precluded the visibility of a line connecting the transversals. Hence, it is highly improbable that the maximal reduction of the effect with medium densities could be due to the perception of a subjective contour.

From both an empirical and a phenomenological point of view, it is interesting to observe that, if we consider the Poggendorff illusion as an instance of the Oppel-Kundt illusion, the latter shows a maximum of the effect when the number of the divisory elements is intermediate with respect to the space to fill (Obonai, 1933; Oyama, 1960; Piaget \& Osterrieth, 1953), that is, a maximum expansion of the space corresponding to a minimum of the Poggendorff effect. This led us to consider the Poggendorff illusion as an illusion of linear extent more than an illusion of direction, and encouraged us to find a general model of the space dynamics. If we compare the data of Experiment 1 with those we obtained in previous research by using parallel-line textures (Masini et al., 1992), we can observe that the minimum of the illusion, $3.42 \mathrm{~mm}$ in the present case, corresponds to the value obtained with a horizontal line texture, $3.544 \mathrm{~mm}$, in Masini et al. The overlapping of these data seems to confirm the hypothesis of an expansion of the space between the verticals. In the first case, it is a function of the texture density; in the second, it is a result of the horizontal lines counterbalancing the apparent elongation of the vertical axis or the con- 
traction of the unfilled space between the verticals. Besides the empirical results discussed above, substantial evidence of the expansion of the space between the verticals derives from the data provided by Experiment 2, by using the same texture configurations of Experiment 1.

It is important to note that Rentschler, Hilz, Sütterlin, and Noguchi (1981), studying the Oppel-Kundt illusion (named the Botti illusion in their work), found that the illusory effect is a U-shaped function of the number of divisory lines. These authors aimed to evaluate the possible role of feature detectors in illusions of filled lateral and angular extent. They used the Botti illusion and the Helmholtz expansion figure, in which an angle appears larger when it contains a fan of lines. They presented evidence that the Helmholtz illusion is fully accounted for by perceptual overestimation of acute angles and can be explained by the interactions of orientation detectors. On the contrary, the same mechanism cannot explain the overestimation of the filled lateral extent in the Botti illusion, because changing the retinal separation between the parallel lines, and thus changing the strength of the lateral inhibition, does not modify the magnitude of the illusion. As mentioned above, they obtained a U-shaped function relating the number of divisory lines to the magnitude of the illusion-with a maximum by using a total of 9 lines and a minimum with 2 and 41 lines. This function is strongly similar to the function we obtained in the present experiments. It is significant that the data of Rentschler et al. showed that the strength of the Botti illusion, in contrast with the angular extent illusion, is independent of the width of the target. The authors state that this phenomenon could depend on a global computation, in which the brain identifies neural detectors activated by endpoints of a distance and a size-constancy mechanism interprets the retinal distances as lengths. The width of the Botti figure is assessed by adding the distance elements between adjacent lines. Thus, the computation would be a global one depending upon information on the size of the target. Given the similarity of the space dynamics studied in both the present experiments and Rentschler et al.'s research, it is possible that the same neural mechanism underlies the space expansion effect that we found.

As mentioned in the introduction, we do not claim that the expansion of the space between the verticals causes the Poggendorff illusion, but merely that the effect is influenced by this expansion. As Wenderoth (1981) pointed out, the manipulation of a variable that affects the magnitude of an illusion does not necessarily imply that this variable, rather than some others, produces the effect in the first instance. Referring to the experiments performed by Brigell and Uhlarik (1980), Wenderoth showed that all the results they obtained could be explained in terms of variables extraneous to the basic mechanisms of the Poggendorff illusion. Wenderoth's observations are indeed relevant; however, it must be remarked that the variable we used to define different experimental conditions does not modify the original Poggendorff configuration.

Even though it cannot be claimed that the Poggendorff illusion depends uniquely on the apparent modification of the space between the verticals, the model presented in the present work provides both a simple explanation of the experimental effect and a quantitative prediction of the strength of the illusion. The results of the simulation (see Figure 5) show that there is a good agreement between the experimental and predicted data. A further advantage of the present approach is that similar models could, in principle, be elaborated so as to quantitatively explain other illusory effects such as the Oppel-Kundt and rectangles illusions. In this respect, our approach can be considered a useful tool for investigating the dynamic properties of perceptual space.

\section{REFERENCES}

Bazzeo, A., Vicario, G. B., \& Zambianchi, E. (1993). Ilusione di Poggendorff: larghezza apparente dello spazio tra le parallele ed entità della distorsione fenomenica. Giomale Italiano di Psicologia, 20, 53-67.

BAZZEO, A., \& ZANuTtini, L. (1978). Illusory dilatation of square surfaces. Perception, 7, 467-471.

BENUSSI, V. (1914). Gesetze der inadäquäten Gestaltauffassun. Archiv für die Gesamte Psycologie, 32, 396-419.

Brigell, M., \& Uhlarik, J. (1980). Bending the parallels of the Poggendorff figure. Bulletin of the Psychonomic Society, 16, 1-4.

Chiang, C. (1968). A new theory to explain geometrical illusions produced by crossing lines. Perception \& Psychophysics, 3, 174-176.

COREN, S., \& GIRGUS, J. S. (1978). Seeing is deceiving: The psychology of visual illusions. Hillsdale NJ: Erlbaum.

DAY, R. H. (1989). Natural and artificial cues, perceptual compromise and the basis of veridical and illusory perception. In D. Vickers \& P. L. Smith (Eds.), Human information processing: Measures, mechanisms, and models (pp. 107-129). Amsterdam: Elsevier Science Publishers.

DAY, R. H., \& Dickinson, R. G. (1976). The components of the Poggendorff illusion. British Journal of Psychology, 67, 537-552.

DAY, R. H., Dickinson, R. G, , \& JoRy, M. K. (1977). The Poggendorff illusion with subjective contours. Quarterly Journal of Experimental Psychology, 29, 219-226.

DAY, R. H., Jolly, W. J., \& DUFFY, F. M. (1987). No evidence for apparent extent between parallels as the basis of the Poggendorff effect. Perception \& Psychophysics, 42, 561-568.

DAY, R. H., \& KASPERCZYK, R. T. (1985). Apparent displacement of lines and dots in a parallel-line figure: A clue to the basis of the Poggendorff effect. Perception \& Psychophysics, 38, 74-80.

de Marco, A., Magnetti, G., \& Saroldi, A. (1991). Poggendorff illusion: Approach to quantitative study of illusion. Proceedings of Cybernetics \& Biophysics Italian Conference, 31, 313-323.

ERIKsson, E. S. (1970). A field theory of visual illusions. British Journal of Psychology, 61, 451-466.

Finlay, D. C., \& Caelli, T. M. (1975). The Poggendorff illusion and estimates of transverse extent. Perceptual \& Motor Skills, 41, 143-148.

GILLAM, B. (1971). A depth processing theory of the Poggendorff illusion. Perception \& Psychophysics, 10, 211-216.

Gillam, B. (1980). Visual illusions. Scientific American, 242, 102-112.

Gillam, B. (1981). False perspective. Perception, 10, 313-318.

GLASs, L. (1970). Effect of blurring on perception of a simple geometric pattern. Nature, 228, 1341-1342.

Goldstein, M. B., \& Weintraub, D. J. (1972). The parallel-less Poggendorff: Virtual contours put the illusion down but not out. Perception \& Psychophysics, 11, 353-355.

Green, R. T., \& Hoy le, E. M. (1963). The Poggendorff illusion as a constancy phenomenon. Nature, 200, 611-612.

GreEN, R. T., \& HoY LE, E. M. (1964). The influence of spatial orientation on Poggendorff illusion. Acta Psychologica, 22, 348-366.

GREENE, E. (1987). The relative contribution of contact and target lines in the magnitude of the Poggendorff effect. Perception, 16, 385-388.

Greene, E., \& Al-Quaddoomi, S. (1990). Use of segment arrays to 
evaluate the strength of angular induction. Perception \& Psychophysics, 47, 243-252.

Greene, E., \& Pavlov, G. (1989). Angular induction as a function of contact and target orientation. Perception, 18, 143-154.

Gregory, R. L. (1972). Cognitive contours. Nature, 238, 51.

Greist-Bousquet, S., \& SChiffman, H. R. (1981). The Poggendorff illusion: An illusion of linear extent? Perception, 10, 155-164.

Greist-Bousquet, S, , \& Schiffman, H. R. (1985). Poggendorff and Müller-Lyer illusions: Common effects. Perception, 14, 427-447.

Greist-Bousquet, S., \& Schiffman, H. R. (1986). The basis of the Poggendorff effect: An additional clue for Day and Kasperczyk. Perception \& Psychophysics, 39, 447-448.

Greist-Bousquet, S., Schiffman, H. R., Dorsett, R., \& Davis, J. (1989). A relationship between apparent extent and alignment in the Poggendorff illusion. Bulletin of the Psychonomic Society, 27, 556-558.

HeLmHoltz, H. L. F. voN (1886). Handbuch der physiologischen Optik (2nd ed.). Leipzig: Voss.

HoTOPF, W. H. N. (1981). Mistracking in alignment illusions. Journal of Experimental Psychology: Human Perception \& Performance, 7, 1211-1246.

Hotopf, W. H. N., \& Ollerearnshaw, C. (1972a). The regression to right angles tendency and the Poggendorff illusion: I. British Journal of Psychology, 63, 359-367.

Hotopf, W. H. N., \& Ollerearnshaw, C. (1972b). The regression to right angles tendency and the Poggendorff illusion: II. British Journal of Psychology, 63, 369-379.

Hotopf, W. H. N., Ollerearnshaw, C., \& Brown, S. (1974). The regression to right angles tendency and the Poggendorff illusion: III. British Journal of Psychology, 65, 213-231.

JudD, C. H. (1899). A study of geometrical illusions. Psychological Review, 6, 241-261.

Masini, R., Sciaky, R., \& Pascarella, A. (1992). The orientation of a parallel-line texture between the verticals can modify the strength of the Poggendorff illusion. Perception \& Psychophysics, 52, 235-242.

MORI, T. (1981). Reduction of the Poggendorff effect by the motion of oblique lines. Perception \& Psychophysics, 29, 15-20.

Newman, C. V. (1973). Variations in size judgements as a function of surface texture. Quarterly Journal of Experimental Psychology, 25, $260-264$.

OBONAI, T. (1933). Contribution to the study of psychophysical induction: III. Experiments on the illusion of filled space. Japanese Journal of Psychology, 8, 699-720.

OYAMA, T. (1960). Japanese studies on so-called geometrical-optical illusions. Psychologia, 3, 7-20.

Parks, T. E., \& HuI, L. (1989). Pictorial depth and the Poggendorff illusion. Perception \& Psychophysics, 46, 465-468.
Piaget, J., \& Osterrieth, P. A. (1953). L'evolution de l'illusion d'Oppel-Kundt en function de l'age. Archives de Psychologie, XXXIV, 133, 1-38.

Pressey, A. W. (1971). An extension of assimilation theory to illusions of size, area, and direction. Perception \& Psychophysics, 9 , 172-176.

Pressey, A. W., \& SWeeney, O. (1972). Some puzzling results on the Poggendorff illusion. Perception \& Psychophysics, 12, 433-437.

QuiNA, K., \& POLLACK, R. H. (1973). Attraction of parallels as a function of intercontour distance. Perceptual \& Motor Skills, 36, 934.

Quina-Holland, K. (1977). Spatial distortions within the Poggendorff figure and its variants: A parametric analysis. Perception \& Psychophysics, 21, 118-124.

Rentschler, I., Hilz, R., Sütterlin, C., \& Noguchi, K. (1981). Illusion of filled lateral and angular extent. Experimental Brain Research, 44, 154-158.

SCHILlER, P., \& WeINER, M. (1972). Binocular and stereoscopic viewing of geometric illusion. Perceptual \& Motor Skills, 13, 739-747.

SPRINGBETT, B. M. (1961). Some stereoscopic phenomena and their implications. British Journal of Psychology, 52, 105-109.

Stuart, G. V., DAY, R. H. (1980). The effect of blurring on intersecting-line illusions. Perception, 9, 537-548.

ThierY, A. (1896). Über geometrisch-optische Täuschungen. Philosophische Studien, 12, 67-126.

Tong, L., \& Weintraub, D. J. (1974). Contour displacements and tracking errors: Probing 'twixt Poggendorff parallels. Perception \& Psychophysics, 15, 258-268.

TYler, C. W., \& Nakayama, K. (1984). Size interactions in the perception of orientation. In L. Spillmann \& B. R. Wooten (Eds.), Sensory experience, adaptation, and perception (pp. 529-546). Hillsdale, $\mathrm{NJ}$ : Erlbaum.

Weintraub, D. J., \& Krantz, D. H. (1971). The Poggendorff illusion: Amputations, rotations, and other perturbations. Perception \& Psychophysics, 10, 257-264.

Wenderoth, P. (1981). Bending the Poggendorff parallels and the rules of inference: A note on Brigell and Uhlarik (1980). Perception \& Psychophysics, 29, 283-284.

WILson, A. E., \& Pressey, A. W. (1976). The role of apparent distance in the Poggendorff illusion. Perception \& Psychophysics, 20, 309-316.

Zanuttini, L. (1976). A new explanation for the Poggendorff illusion. Perception \& Psychophysics, 20, 29-32.

(Manuscript received December 7, 1992; revision accepted for publication September 22, 1993.) 